\title{
Mitteilungen aus der Gesellschaft für afrikanisches Recht (2017)
}

\author{
Harald Sippel $^{*}$
}

Wie bereits in bisherigen Ausgaben unserer Zeitschrift geschehen, ${ }^{1}$ werden die Mitglieder der Gesellschaft für afrikanisches Recht e.V. und die interessierte Öffentlichkeit auch für das Jahr 2017 über die Tätigkeiten des Vorstandes sowie die Jahrestagung informiert und auf die geplanten Veranstaltungen unserer wissenschaftlichen Vereinigung hingewiesen.

\section{Jahrestagung 2017}

Die 44. Jahrestagung der Gesellschaft für afrikanisches Recht wurde gemeinsam mit dem Arnold-Bergstraesser-Institut an der Universität Freiburg im Breisgau, Deutschland, am 10. und 11. November 2017 zum Thema „Armed Conflict in Africa: Legal and Political Measures for Protecting Displaced People and Preventing Forced Migration" veranstaltet.

Nach der Eröffnung der Tagung durch den Direktor des gastgebenden Arnold-Bergstraesser-Instituts, Herr Prof. Dr. Andreas Mehler, die Vertreterin der Stiftung Entwicklungszusammenarbeit Baden-Württemberg, Frau Joyce Muvunji, und den Verfasser dieses Berichts sprachen am ersten Konferenztag Frau Dr. Nneka Okechukwu (Max Planck Foundation for International Peace and the Rule of Law, Heidelberg, Deutschland) über „The Evolution of the Responsiblity to Protect“, Herr Prof. Dr. Dennis Dijkzeul (Ruhr-Universität Bochum, Deutschland) zu „The Contested Principles of Humanitarianism“, Frau Dr. Carolien Jacobs (Universität Leiden, Niederlande) über „Justice for Internally Displaced Persons in the Democratic Republik of Congo“ und Herr Dr. Aimé-Parfait Niyonkuru (Arnold-BergstraesserInstitut, Freiburg im Breisgau, Deutschland) zu „The Judicial Protection of Citizens in Postconflict Countries: The Case of Burundi“. Der erste Konferenztag endete mit einer angeregten Podiumsdiskussion, die mit dem Thema „The Protection of Displaced Persons in Africa: Promises and Pitfalls“ das Leitmotiv der Tagung besonders herausstellte.

Am zweiten Veranstaltungstag präsentierte zunächst Herr Dr. Oliver Meinecke (Bundesministerium für wirtschaftliche Zusammenarbeit und Entwicklung, Berlin, Deutschland) seinen Vortrag zu „Supporting Refugees in Contexts of Fragility - Approaches by German Development Cooperation“"vor, gefolgt von Frau Dr. Marina Sharpe (McGill Universität, Kanada), die über „The Regional Law of Refugee Protection in Africa“" referierte. Einen ge-

* Privatdozent Dr. Harald Sippel ist Vorsitzender des Vorstandes der Gesellschaft für afrikanisches Recht e.V. (E-Mail: harald.sippel@rechtinafrika.de).

1 Sippel, Harald, Mitteilungen aus der Gesellschaft für afrikanisches Recht (2016), in: Recht in Afrika 19 (2016), S. 245-247, und Mitteilungen aus der Gesellschaft für afrikanisches Recht (2015), in: Recht in Afrika 18 (2015), S. 264-266, sowie Mitteilungen aus der Gesellschaft für afrikanisches Recht (2014), in: Recht in Afrika 17 (2014), S. 237-242. 
lungenen Abschluss der Tagung bildeten die „Perspectives of East African Legal Practitioners" zum Tagungsthema, welche von Herrn Alexis Manirakiza (Burundi), Herrn Claude Mwanza Wa Kalombo (Demokratische Republik Kongo), Herrn Jean Claude Nsengiyumva (Ruanda), Herrn Bernard Ntahiraja (Burundi) und Frau Elizabeth Simon Swai (Tansania) vorgestellt wurden.

Gedankt sei den Referenten sowie allen Gästen, welche die Diskussionen durch ihre Wortbeiträge bereichert haben. Besonders dankbar sind wir dem Arnold-Bergstraesser-Institut, namentlich Herrn Professor Mehler und Frau Dr. Franzisca Zanker sowie ihren Mitarbeiterinnen und Mitarbeitern für die hervorragende Organisation der Kooperationstagung. Unser herzlicher Dank gilt der Stiftung Entwicklungszusammenarbeit Baden-Württemberg, ohne deren großzügige finanzielle Unterstützung die Ausrichtung der Tagung nicht möglich gewesen wäre.

\section{Vorstand}

Die Mitgliederversammlung wählte während der Jahrestagung 2017 in Freiburg im Breisgau den neuen Vorstand der Gesellschaft für afrikanisches Recht für die Kalenderjahre 2018 und 2019, der sich wie folgt zusammensetzt:

- Vorstandsvorsitz - Harald Sippel

- Stellvertretender Vorsitz - Oliver Meinecke

- Generalsekretär - Jörg Kleis

- Finanzen - Dirk Otto

- Tagungen - Katrin Seidel

- Zeitschrift - Hartmut Hamann

- Öffentlichkeitsarbeit - Hatem Elliesie

- Internetseite - Wieland Lehnert

- Vernetzung im südlichen Afrika - Oliver Ruppel

- Vernetzung mit internationalen Organisationen - Thilo Marauhn

- Vernetzung mit Menschenrechtsorganisationen - Prosper Maguchu

Den ausgeschiedenen Vorstandsmitgliedern, Kimon Haars und Ulrike Wanitzek, die sich nicht mehr zur Wahl gestellt hatten, sei für ihre langjährige hervorragende Mitarbeit im Vorstand sehr herzlich gedankt.

\section{Neue Geschäftsstelle}

Herr Dr. Jörg Kleis nimmt sich als neuer Generalsekretär der Gesellschaft für afrikanisches Recht fortan der Betreuung der Mitglieder an. Mit der Übernahme des Generalsekretariats hat sich auch die Anschrift der Geschäftsstelle des Vereins geändert. Sie lautet nunmehr: Gesellschaft für afrikanisches Recht e.V., c/o Dr. Jörg Kleis, Choriner Str. 83, 10119 Berlin, Deutschland (gs@rechtinafrika.de). 


\section{Publikationen und Internetseite}

Das vorliegende Heft von „Recht in Afrika“ vervollständigt bereits den 20. Jahrgang unserer Zeitschrift. Sie verfolgt das Ziel, ein Informations- und Diskussionsforum für die unterschiedlichen und vielfältigen Ausprägungen von Recht, das auf dem afrikanischen Kontinent zur Anwendung kommt, zu sein. Es wird sehr herzlich dazu eingeladen, der Redaktion der Zeitschrift publikationsfähige Beiträge zum afrikanischen Recht (Artikel, Berichte, Buch- und Urteilsbesprechungen) anzubieten.

Informationen über die Tätigkeiten und Veranstaltungen der Gesellschaft für afrikanisches Recht e.V. sind im Internet unter www.rechtinafrika.de erhältlich.

\section{Internationale Kooperation}

Das von der Robert-Bosch-Stiftung (Stuttgart, Deutschland) geförderte und von der Gesellschaft für afrikanisches Recht begleitete Austauschprogramm für berufserfahrene junge Juristen aus ost- und zentralafrikanischen Staaten mit der Justiz in Baden-Württemberg und Rheinland-Pfalz wurde im laufenden Jahr fortgeführt. Über eine im Rahmen dieses Programms veranstaltete Podiumsdiskussion informiert der Bericht von Herrn Aniello Ambrosio in diesem Heft. ${ }^{2}$

\section{Jahrestagung 2018}

Die 45. Jahrestagung der Gesellschaft für afrikanisches Recht soll in Kooperation mit der Juristischen Fakultät der Westfälischen Wilhelms-Universität in Münster, Deutschland, zum Thema „Wirtschaftliche Integration afrikanischer Staaten und Freihandelsabkommen” (Arbeitstitel) am Freitag, dem 9. November 2018, und am Samstag, dem 10. November 2018, stattfinden. Nähere Informationen werden zu gegebener Zeit den Mitgliedern der Gesellschaft und auf der Homepage unseres Vereins unter www.rechtinafrika.de mitgeteilt.

2 Unter dem Titel Bericht über die Podiumsdiskussion „,Die Aufklärung von Straftaten gegen das Völkerrecht als Voraussetzung für den Aufbau einer effizienten Justiz" in Stuttgart am 9. November 2017. 


\section{Impressum}

Recht in Afrika - Law in Africa - Droit en Afrique - Zeitschrift der Gesellschaft für afrikanisches Recht

Herausgeber: Gesellschaft für afrikanisches Recht e.V., vertreten durch den Vorsitzenden des Vorstandes, Herrn Dr. Harald Sippel, Barbarossastraße 31, 09112 Chemnitz

Schriftleitung: RA Prof. Dr. Hartmut Hamann, Falkertstraße 82, D-70193 Stuttgart Telefon: +49 (o) 711 120950-30 | Fax : +49 (o) 711 120950-50

Mail:hamann@hamann-legal.de

Erscheinungsweise: 2 Ausgaben pro Jahr

Druck und Verlag: Nomos Verlagsgesellschaft mbH \& Co. KG - Waldseestr. 3-5 D-76530 Baden-Baden. Telefon +49-7221-2104-0 / Fax 49-7221-2104-27

E-Mail:nomos@nomos.de

Anzeigen: sales friendly Verlagsdienstleistungen - Inh. Frau Bettina Roos - Pfaffenweg 15 - D-53227 Bonn Telefon +49-228-97898-o / Fax +49-228-97898-20 E-Mail: roos@sales-friendly.de

Urheber- und Verlagsrechte: Die Zeitschrift und alle in ihr enthaltenen einzelnen Beiträge sind urheberrechtlich geschützt. Jede Verwertung außerhalb der engen Grenzen des Urheberrechtsgesetzes ist ohne Zustimmung des Verlages unzulässig. Das gilt insbesondere für Vervielfältigungen, Übersetzungen, Mikroverfilmungen und die Einspeicherung und Verarbeitung in elektronischen Systemen. Der Nomos Verlag beachtet die Regeln des Börsenvereins des Deutschen Buchhandels e.V. zur Verwendung von Buchrezensionen.

ISSN 2363-6270

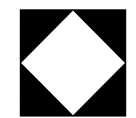

Nomos 